\title{
The value of PET/CT in determining lymph node metastasis of endometrial cancer
}

\author{
Emine Budak, Ahmet Yanarateş \\ University of Health Sciences, Izmir Dr. Suat Seren Chest Diseases and Surgery Training and Research Hospital, \\ Department of Nuclear Medicine, Turkey
}

\begin{abstract}
Objectives: In our study, the role of fluorine-18 fluorodeoxyglucose positron emission tomography/computed tomography (F-18 FDG PET/CT) in determining lymph node metastasis of endometrial cancer was evaluated.

Material and methods: The present retrospectively registered study included 80 patients with endometrial cancer who underwent PET/CT in preoperative period. The patients underwent total hysterectomy with bilateral salpingo-oophorectomy and lymph node dissection. Lymph node status was evaluated in histopathologic examination and PET/CT imaging and, the results were compared.

Results: There were 80 patients (mean age 62 years) in the present study. Lymph node metastasis was detected in five patients, whereas 75 patients had no lymph node metastasis. A total of 1,471 lymph nodes were examined histopathologically, revealing benign findings in 1,433 (pelvic $=1018$, paraaortic $=415$ ) and malignant findings in 38 (pelvic $=28$, paraaortic $=10$ ) lymph nodes. The accuracy, sensitivity and specificity of PET/CT in determining lymph node metastasis was $95 \%, 80 \%$ and $96 \%$ in patient-based evaluation, and $97.4 \%, 78.9 \%$ and $98.6 \%$ in lymph node-based evaluation, respectively. The detection sensitivity of PET/CT was $0 \%, 81.4 \%$ and $100 \%$ in metastatic lymph nodes with a short diameter of $\leq 4 \mathrm{~mm}, 5-9 \mathrm{~mm}$ and $\geq 10 \mathrm{~mm}$, respectively. PET/CT could detect $73.3 \%$ of metastatic lymph nodes that had $<10 \mathrm{~mm}$ short diameter.

Conclusions: PET/CT is useful method in detecting lymph node metastasis especially that are disregarded by CT or MR in endometrial cancer. Although PET/CT doesn't fully replace the surgical staging, its utilization in preoperative period may guide surgical procedure.
\end{abstract}

Key words: FDG PET/CT; endometrial cancer; lymph node metastasis

Ginekologia Polska 2019; 90, 10: 565-570

\section{INTRODUCTION}

The most common gynecological neoplasm in industrialized countries is endometrial cancer [1]. The International Federation of Gynecology and Obstetrics (FIGO) recommends total hysterectomy with bilateral salpingo-oophorectomy and lymph node dissection for surgical staging of endometrial cancer [2,3]. Prognosis in such patients is affected by numerous factors, such as tumor histology, grade, lymph node metastasis, depth of myometrial invasion, cervical invasion, lymphovascular invasion and patient's age [4, 5]. Pelvic lymph nodes are a common site of involvement in endometrial cancer, and patients with lymph node metastasis have considerably lower survival than patients without nodal metastasis $[6,7]$. Randomized studies suggest that a routine systematic pelvic lymphadenectomy may contribute to surgical staging without any effect on survival in early-stage endometrial cancer $[8,9]$. In this regard, the major role of lymphadenectomy is to decide on adjuvant therapy by contributing to the staging and the prediction of prognosis in endometrial cancer [10]. Lymph node dissection may cause significant morbidity, and metastasis may not be found in patients undergoing dissection [10]. Thus, the accurate prediction of lymph node status in preoperative period is important if futile lymph node dissection is to be avoided [11]. Magnetic resonance (MR) and computed tomography (CT) may fail to determine lymph node metastasis accurately $[12,13]$. Fluorine-18 fluorodeoxyglucose positron emission tomography/computed tomography (F-18 FDG $\mathrm{PET} / \mathrm{CT}$ ) is a commonly used method for the diagnosis and staging of numerous malignancies, and in evaluating response to therapy, providing both functional and anatomical information. It is suggested that functional changes that are detectable on a PET/CT precede morphological changes that can be detected using conventional CT and MR [14]. 


\section{Objectives}

The authors of the present study assess the role of F-18 FDG PET/CT in determining lymph node metastasis of endometrial cancer.

\section{MATERIAL AND METHODS}

Patients

This retrospective study included 80 patients with a histopathological diagnosis of endometrial cancer who underwent preoperative F-18 FDG PET/CT in our department between February 2010 and March 2014.

\section{Surgery}

All patients underwent total hysterectomy with bilateral salpingo-oophorectomy and pelvic lymphadenectomy (external iliac, superficial and deep common iliac lymph nodes). There were positive lymph nodes in the pelvic and/or paraaortic areas on PET/CT scans in 7 patients. Furthermore, there were 43 patients with suspected for paraaortic lymph node metastasis during surgery. Therefore, these fifty patients underwent an additional paraaortic lymphadenectomies (precaval and paracaval, superficial and deep interaortocaval and paraaortic lymph nodes up to the renal vessels). All lymph nodes were evaluated by intraoperative inspection and palpation. Palpable, enlarged or fixed lymph nodes were regarded suspicious for malignancy. Furthermore, frozen section of uterine resection was evaluated in terms of tumor size, grade, histology and myometrial and cervical invasion status. Patients with myometrial invasion $<50 \%$ and without cervical invasion endometrioid cancer (grade 1 or tumor diameter $<2 \mathrm{~cm}$ grade 2 ) were considered to be at low risk for paraaortic lymph node metastasis, whereas others high risk. Paraaortic lymphadenectomy was performed in high-risk patients and those with suspicious lymph nodes during the operation.

There was a patient who was suspected to have omental metastasis during the operation, so she underwent omental resection. Although no metastasis was detected in frozen sections of the omentum, microscopic metastasis was found in the final histopathological evaluation. Since the distant metastasis of the patient with stage 4B could not be detected during the operation or preoperative PET/CT, lymphadenectomy was performed.

\section{Histopathologic Evaluation of Lymph Nodes}

Uterus, bilateral adnexal tissues, lymph nodes and any other resected tissues were analysed by experienced pathologists.

Lymph nodes were fixed with $10 \%$ formalin and then embedded in paraffin. Paraffin blocks were sliced and processed. Tissue sections were firstly stained with hematoxylin and eosin $(\mathrm{H} \& \mathrm{E})$. If metastasis was detected in this proce- dure, no further histological examination was performed. If the serial $\mathrm{H} \& \mathrm{E}$ sections were negative, immunohistochemical (IHC) methods were performed. PAN-cytokeratin was used for IHC evaluation. Histopathologically, the lymph nodes were defined as benign and malignant.

\section{F-18 FDG PET/CT}

PHILIPS GEMINITF 16 Slice PET/CT scan was used for imaging. Patients with a blood glucose level below $200 \mathrm{mg} / \mathrm{dL}$ following an at least 6 hours of fasting received an intravenous 8-11 mCi FDG injection. The urinary bladder was evacuated approximately 60 minutes after the injection, and the body area from the vertex of the skull to the upper femur was scanned. CT images were captured initially (140 kV, $100 \mathrm{mAs}, 5 \mathrm{~mm}$ slice) followed by PET scans. PET scanning was performed at 9-10 bed positions depending on the height of the patient, each bed position lasting 90 seconds.

The blood pool activity was taken into consideration while evaluating the FDG uptake by the lymph nodes on PET/CT. Regardless of the lesion size, a lymph node showing a FDG uptake equal to or below the blood pool activity was considered negative for malignancy, and a FDG uptake above this threshold was considered positive for malignancy.

\section{Data and Statistical Analyses}

The histopathological examination results were considered as the reference standard in the evaluation of lymph node metastasis. A histopathological examination of the lymph nodes identified the lymph nodes as benign or malignant. The result of PET/CT was considered a true negative (TN) if it showed negative findings, and the result was considered false positive (FP) if the PET/CT showed positive findings in a histopathologically benign lymph node. The result of PET/CT was considered a true positive (TP) if it showed positive findings, and the result was considered a false negative (FN) if it showed negative findings in a histopathologically malignant lymph node. The accuracy, sensitivity and specificity of PET/CT in determining lymph node metastasis was calculated using a standard formula on a patient and lymph node basis.

$$
\begin{aligned}
& \text { Sensitivity }=T P /(T P+F N), \text { Specificity }=T N /(T N+F P), \\
& \text { Accuracy }=(T P+T N) /(T P+F P+T N+F N)
\end{aligned}
$$

\section{RESULTS}

\section{Patients and Histopathologic Findings}

The study included 80 patients who had endometrial cancer with a mean age of $62 \pm 5.4$ years (range 37-80). All patients underwent a pelvic lymphadenectomy, while 50 patients underwent an additional paraaortic lymphadenectomy. Lymph node metastasis was detected in five patients, whereas 75 patients had no lymph node metastasis. 
Of the patients with lymph node metastasis, one had endometroid and 4 had nonendometroid (mixed $=2$, serous $=1$, undifferentiated $=1$ ) histology. The tumor grade was 2 in 1 patient and 3 in 4 patients. According to FIGO 2009 staging, 2 of the patients were in stage $3 C 1,2$ of them were in stage $3 C 2,1$ of them was in stage $4 B$.

In patients without lymph node metastasis the histologic types were endometrioid carcinoma $(n=47)$, adenocarcinoma with squamous differentiation $(n=10)$, squamous carcinoma ( $n=8)$, undifferentiated carcinoma $(n=6)$, serous carcinoma $(n=2)$, clear cell carcinoma $(n=1)$ and mucinous carcinoma $(n=1) .22$ cases were grade 1,32 cases were grade 2 and 21 cases were grade 3 . Of the 75 patients, 40 were stage $1 A, 12$ were stage $1 B, 10$ were stage 2,5 were stage $3 \mathrm{~A}$ and 8 were stage $3 \mathrm{~B}$.

When evaluated on an lymph node basis, of the total 1,471 lymph nodes, 1,046 were found in the pelvic area and 425 in the paraaortic area in a histopathological examination. Of these lymph nodes, 1,433 (pelvic lymph node $=1018$, paraaortic lymph node $=415$ ) were benign and 38 (pelvic lymph node $=28$, paraaortic lymph node $=10$ ) were malignant $($ Tab. 1$)$.

\section{Lymph Node Findings on PET/CT}

Lymph node metastasis was detected in five out of 80 patients, whereas 75 patients had no lymph node metastasis. When evaluated on a patient basis, PET/CT was negative in 72 (TN) and positive (FP) in three of the 75 patients with a benign lymph node. $\mathrm{PET} / \mathrm{CT}$ was positive in four (TP) and negative (FN) in one out of the five patients with at least one malignant lymph node on a histopathological examination. The results are presented in Table 2 . When evaluated on a patient basis, the accuracy, sensitivity and specificity of PET/CT in detecting lymph node metastasis was $95 \%, 80 \%$ and, $96 \%$ respectively.

\begin{tabular}{|l|l|l|l|}
\hline Table 1. Histopathologically evaluated lymph nodes \\
\hline & Benign & Malign & Total \\
\hline Pelvic lymph node & 1018 & 28 & 1046 \\
\hline Paraaortic lymph node & 415 & 10 & 425 \\
\hline Total & 1433 & 38 & 1471 \\
\hline
\end{tabular}

Results are shown as number

\begin{tabular}{|c|c|c|c|}
\hline & PET/CT positive & PET/CT negative & Total \\
\hline Pathology positive & 4 & 1 & 5 \\
\hline Pathology negative & 3 & 72 & 75 \\
\hline Total & 7 & 73 & 80 \\
\hline
\end{tabular}

Results are shown as number

\begin{tabular}{|c|c|c|c|c|}
\hline & & $\begin{array}{l}\text { PET/CT } \\
\text { positive }\end{array}$ & $\begin{array}{l}\mathrm{PET} / \mathrm{CT} \\
\text { negative }\end{array}$ & Total \\
\hline \multirow{2}{*}{$\begin{array}{l}\text { Pelvic lymph } \\
\text { node }\end{array}$} & pathology positive & 22 & 6 & 28 \\
\hline & pathology negative & 11 & 1007 & 1018 \\
\hline \multirow{2}{*}{$\begin{array}{l}\text { Paraaortic } \\
\text { lymph node }\end{array}$} & pathology positive & 8 & 2 & 10 \\
\hline & pathology negative & 9 & 406 & 415 \\
\hline Total & & 50 & 1421 & 1471 \\
\hline
\end{tabular}

Results are shown as number

When evaluated on an lymph node basis, PET/CT was positive in 20 (FP) and negative in 1,413 (TN) out of the 1,433 lymph nodes that qualified histopathologically as benign. PET/CT was positive in 30 (TP) and negative in eight (FN) of the 38 lymph nodes that qualified as malignant upon a histopathological examination. The results are presented in Table 3. When evaluated on an lymph node basis, the accuracy, sensitivity and specificity of PET/CT in detecting lymph node metastasis was $97.4 \%, 78.9 \%$ and $98.6 \%$, respectively. Sample lymph node findings of PET/CT that were evaluated based on histopathological results are presented in Figure 1.

When 38 metastatic lymph nodes are grouped according to their short diameters; 3 of them were $\leq 4 \mathrm{~mm}, 27$ were 5-9 mm, and 8 were $\geq 10 \mathrm{~mm}$. PET/CT could not detect any metastatic lymph nodes with a short diameter of $\leq 4 \mathrm{~mm}$, while it was true positive in 22 of those with a short diameter of 5-9 $\mathrm{mm}$ and in all of those with $\geq 10 \mathrm{~mm}$. Thus, PET/CT was able to detect 22 of 30 metastatic lymph nodes with a short diameter of less than $10 \mathrm{~mm}$ (73.3\%). The detection sensitivity of PET/CT was $0 \%(0 / 3), 81.4 \%(22 / 27)$ and $100 \%$ in metastatic lymph nodes with a short diameter of $\leq 4 \mathrm{~mm}$, 5-9 $\mathrm{mm}$ and $\geq 10 \mathrm{~mm}$, respectively.

\section{DISCUSSION}

Detecting the presence of lymph node metastasis in endometrial cancer is important in treatment decisions, as well as in predicting prognosis. However, only $20-25 \%$ of high-risk patients show nodal metastasis and up to $80 \%$ of patients undergo a futile systematic lymphadenectomy [15]. Furthermore, it has been demonstrated that a routine systematic lymphadenectomy offers no survival benefit in early-stage endometrial cancer $[8,9]$. Lymph node dissection is currently considered the optimum method in determining the status of lymph nodes in endometrial cancer [11], although there are ongoing efforts to identify a preoperative, predictive non-invasive method. Determining the status of lymph nodes preoperatively avoids futile lymph node dissection, thereby decreasing morbidity and reducing costs. CT and MR are commonly used methods in 
A
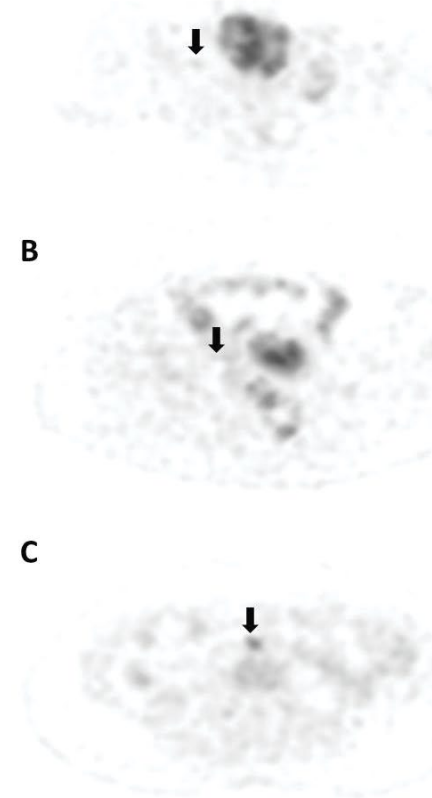

D

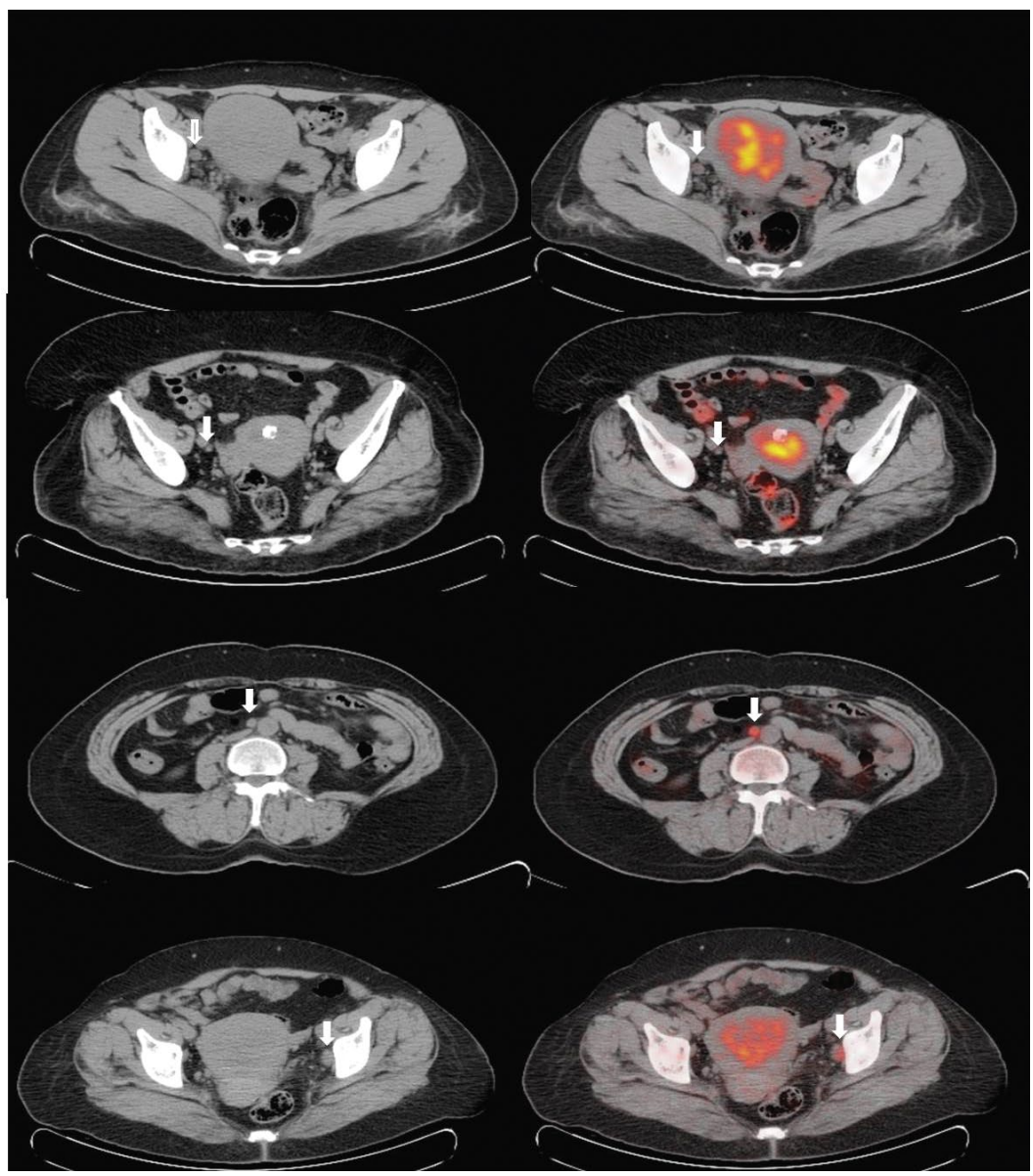

Figure 1. (A-D): Axial PET, $C T$, and fusion images of the lymph nodes evaluated as true negative ( $A$, arrow), false negative (B, arrow), true positive (C, arrow), and false positive (D, arrow) on PET/CT are presented.; A. No lymph nodes with increased FDG uptake were observed in the pelvic or paraaortic regions on the PET/CT of a 44-year-old patient. No metastasis was determined upon a histopathological examination of the patient's 52 lymph nodes (44 pelvic and 8 paraaortic). The PET/CT result was a true negative for all 52 lymph nodes; B. No increased FDG uptake was observed in the lymph nodes seen in the pelvic and paraaortic regions in the PET/CT of a 74-year-old patient. A total of 28 lymph nodes were dissected from the pelvic ( $\mathrm{n}: 21)$ and paraaortic $(\mathrm{n}: 7)$ regions in the case, in which metastasis was suspected intraoperatively. Metastasis was determined in the five lymph nodes in the right external and internal iliac regions, while other lymph nodes were determined to be benign. The PET/CT was false negative in those five lymph nodes and true negative in the other 23 lymph nodes; $\mathbf{C}$. An increased FDG uptake was noted in one lymph node in the paraaortic region and in three lymph nodes in the pelvic region in a PET/CT of a 49-year-old patient. Metastasis was determined positive in the four lymph nodes histopathologically, and hence PET/CT findings were evaluated to be true positive; D. An increased FDG uptake was noted in three lymph nodes in the paraaortic region and in three lymph nodes in the pelvic region on a PET/CT. No metastasis was determined to be positive histopathologically in the total number of 30 lymph nodes (22 pelvic, 8 paraaortic) in this case. The PET/CT findings were considered false positive in six lymph nodes and true negative in the remaining 24 lymph nodes

the preoperative evaluation of patients with endometrial cancer. However, these conventional methods are predicated on the size of the lymph nodes in the evaluation and a short diameter of $\geq 10 \mathrm{~mm}$ is the most accepted criterion for the identification of suspected lymph nodes. On this point, it is inevitable that $<10 \mathrm{~mm}$ short diameter metastatic lymph nodes are underestimated by CT or MR. However, FDG PET/CT provides functional data while also identifying morphological changes, suggesting that it may be superior to anatomical visualization alone in the evaluation of the status of lymph nodes [14]. In the present study, there were 30 metastatic lymph nodes with $<10 \mathrm{~mm}$ short diameter. PET/CT was able to detect 22 of them (73.3\%) and so had a great value in the detection of subcentimeter metastatic lymph nodes which have been disregarded by CT and MR. CT and MR are asserted to have a sensitivity of $29-66 \%$ and a specificity of $73-99 \%$ in various studies $[12,16,17]$. A study involving 287 patients with endometrial cancer found that PET/CT was more sensitive than MR in detecting lymph node metastasis in a patient-based evaluation (70\% vs. 34\%), although specificity rates were similar (95.4\% vs. $95 \%$ ). The sensitivity and specificity of PET/CT were found to be $79.4 \%$ and $96.7 \%$ in an lymph node-based evaluation, with MR showing rates of $51.6 \%$ and $97.6 \%$, respectively [18]. In a meta-analysis of 13 patient-based studies involving a total of 861 patients with endometrial cancer, the 
pooled specificity of FDG PET/CT in detecting lymph node metastasis was $94 \%$ (93-96\%) and the pooled sensitivity was $72 \%(63-80 \%)$ [14]. In another meta analysis of seven studies (243 patients with endometrial cancer), the pooled specificity and sensitivity of PET or PET/CT in detecting lymph node metastasis was $94.7 \%$ (90.4-97.4\%) and 63\% (48.7-75.7\%), respectively [19]. In a study by Crivellaro et al. [15] involving 76 high-risk patients (serous/clear cell carcinoma, grade 2 with deep myometrial invasion, grade 3) with clinical stage 1 endometrial cancer, the accuracy, sensitivity and specificity of PET/CT in detecting lymph node metastasis were $94.7 \%, 78.6 \%$ and $98.4 \%$ in a patient-based evaluation, and $95.8 \%, 67.6 \%$ and $98.2 \%$ in an lymph node-based evaluation, respectively. In a study involving 40 patients with stage $1 \mathrm{~A}-\mathrm{C}$ endometrial cancer, the accuracy, sensitivity and specificity of PET/CT in detecting lymph node metastasis were found to be $97.8 \%, 53.3 \%$ and $99.6 \%$ in a lymph node-based evaluation, respectively. When the lymph nodes were grouped according to their diameters, sensitivity was $16.7 \%, 66.7 \%$ and $93.3 \%$ in lesions measuring $\leq 4 \mathrm{~mm}, 5-9 \mathrm{~mm}$ and $\geq 10 \mathrm{~mm}$ in diameter, respectively [20]. In a study by Kitajima et al. [21] involving patients with endometrial cancer and cervical cancer and using a similar lymph node classification, sensitivity was $12.5 \%, 66.7 \%$ and $100 \%$, respectively. Another study using FDG-PET detected no lymph node metastasis smaller than $6 \mathrm{~mm}$ [22]. The accuracy, sensitivity and specificity of PET/CT in detecting lymph node metastasis in the present study were found to be $95 \%, 80 \%$ and $96 \%$ in a patient-based evaluation, and $97.4 \%, 78.9 \%$ and $98.6 \%$ in an lymph node-based evaluation. Also, the detection sensitivity of PET/CT was $0 \%, 81.4 \%$ and $100 \%$ in metastatic lymph nodes with a short diameter of $\leq 4 \mathrm{~mm}, 5-9 \mathrm{~mm}$ and $\geq 10 \mathrm{~mm}$, respectively. PET/CT could not detect any three metastatic nodes $4 \mathrm{~mm}$ or smaller. This is probably because of the currently used PET/CT techniques cannot detect micrometastatic diseases due to their low spatial resolution. The specificity of PET/CT in the present study was similar to literatüre results and, the sensitivity was higher than some of those reported in literature. The authors consider that lymph nodes evaluated in studies with variable sizes and the inability to recognize small-size lymph node metastasiss may result in different sensitivity rates being reported in such studies. For example, in our study, the rate of metastatic lymph nodes with a short diameter of $\leq 4 \mathrm{~mm}$ was $3 / 38$ whereas in the study by Kitajima it was $16 / 45$. Furthermore, the selection of low- or high-risk patients in some studies may have also affected the results.

\section{CONCLUCIONS}

The present study is limited by its retrospective design and the relatively small number of patients with lymph node metastasis. That said, PET/CT showed high sensitivity, specificity and accuracy in the detection of lymph node metastasis, and had a great value in the detection of subcentimeter ones. Therefore, PET/CT can direct the surgical procedure even though it does not completely replace the surgical staging.

\section{REFERENCES}

1. Signorelli M, Guerra L, Buda A, et al. Role of the integrated FDG PET/CT in the surgical management of patients with high risk clinical early stage endometrial cancer: detection of pelvic nodal metastases. Gynecol Oncol. 2009; 115(2):231-235, doi: 10.1016/j.ygyno.2009.07.020, indexed in Pubmed: 19695685.

2. Pecorelli S. Revised FIGO staging for carcinoma of the vulva, cervix and endometrium. Int J Gynaecol Obstet. 2009; 105(2): 103-104, doi: 10.1016/j.ijgo.2009.02.012, indexed in Pubmed: 19367689.

3. Creasman W. Revised FIGO staging for carcinoma of the endometrium. Int J Gynaecol Obstet. 2009; 105(2): 109, doi: 10.1016/j.ijgo.2009.02.010, indexed in Pubmed: 19345353.

4. Uharcek P, Mlyncek M, Lajtman E, et al. Prognostic factors in endometrial carcinoma. J Obstet Gynaecol Res. 2008; 34(5): 776-783, indexed in Pubmed: 18958927.

5. Colombo N, Creutzberg C, Amant F, et al. ESMO-ESGO-ESTRO Endometrial Consensus Conference Working Group. ESMO-ESGO-ESTRO Consensus Conference on Endometrial Cancer: diagnosis, treatment and follow-up. Ann Oncol. 2016; 27(1): 16-41, doi: 10.1093/annonc/mdv484, indexed in Pubmed: 26634381.

6. Manetta A, Delgado G, Petrilli E, et al. The significance of paraaortic node status in carcinoma of the cervix and endometrium. Gynecol Oncol. 1986; 23(3): 284-290, doi: 10.1016/0090-8258(86)90128-9, indexed in Pubmed: 3957115.

7. Gal D, Recio FO, Zamurovic D, et al. Lymphvascular space involvement-a prognostic indicator in endometrial adenocarcinoma. Gynecol Oncol. 1991; 42(2): 142-145, doi: 10.1016/0090-8258(91)90334-2, indexed in Pubmed: 1894173.

8. Kitchener H, Swart AMC, Qian Q, et al. ASTEC study group. Efficacy of systematic pelvic lymphadenectomy in endometrial cancer (MRC ASTEC trial): a randomised study. Lancet. 2009; 373(9658): 125-136, doi: 10.1016/S0140-6736(08)61766-3, indexed in Pubmed: 19070889.

9. Benedetti Panici P, Basile S, Maneschi F, et al. Systematic pelvic lymphadenectomy vs. no lymphadenectomy in early-stage endometrial carcinoma: randomized clinical trial. J Natl Cancer Inst. 2008; 100(23): 1707-1716, doi: 10.1093/jnci/djn397, indexed in Pubmed: 19033573.

10. MayK, Bryant A, Dickinson HO, etal. Lymphadenectomy for the management of endometrial cancer. Cochrane Database Syst Rev. 2010(1): CD007585, doi: 10.1002/14651858.CD007585.pub2, indexed in Pubmed: 20091639.

11. Park JY, Lee JJ, Choi HJ, et al. The Value of Preoperative Positron Emission Tomography/Computed Tomography in Node-Negative Endometrial Cancer on Magnetic Resonance Imaging. Ann Surg Oncol. 2017; 24(8): 2303-2310, doi: 10.1245/s10434-017-5901-8, indexed in Pubmed: 28550488.

12. Connor JP, Andrews Jl, Anderson B, et al. Computed tomography in endometrial carcinoma. Obstet Gynecol. 2000; 95(5): 692-696, doi: 10.1016/s0029-7844(99)00626-2, indexed in Pubmed: 10775731.

13. Ryoo UiN, Choi $\mathrm{CH}$, Yoon JiY, et al. MR imaging in endometrial carcinoma as a diagnostic tool for the prediction of myometrial invasion and lymph node metastasis. Cancer Res Treat. 2007; 39(4): 165-170, doi: 10.4143/crt.2007.39.4.165, indexed in Pubmed: 19746183.

14. Bollineni VR, Ytre-Hauge S, Bollineni-Balabay O, et al. High Diagnostic Value of 18F-FDG PET/CT in Endometrial Cancer: Systematic Review and Meta-Analysis of the Literature. J Nucl Med. 2016; 57(6): 879-885, doi: 10.2967/jnumed.115.170597, indexed in Pubmed: 26823564.

15. Crivellaro C, Signorelli M, Guerra L, et al. Tailoring systematic lymphadenectomy in high-risk clinical early stage endometrial cancer: the role of 18F-FDG PET/CT. Gynecol Oncol. 2013; 130(2): 306-311, doi: 10.1016/j. ygyno.2013.05.011, indexed in Pubmed: 23707673.

16. Rockall AG, Sohaib SA, Harisinghani MG, et al. Diagnostic performance of nanoparticle-enhanced magnetic resonance imaging in the diagnosis of lymph node metastases in patients with endometrial and cervical cancer. J Clin Oncol. 2005; 23(12): 2813-2821, doi: 10.1200/JCO.2005.07.166, indexed in Pubmed: 15837995.

17. Rockall AG, Meroni R, Sohaib SA, et al. Evaluation of endometrial carcinoma on magnetic resonance imaging. Int J Gynecol Cancer. 2007; 17(1): 188-196, doi: 10.1111/j.1525-1438.2007.00805.x, indexed in Pubmed: 17291252. 
18. Kim HJ, Cho A, Yun M, et al. Comparison of FDG PET/CT and MRI in lymph node staging of endometrial cancer. Ann Nucl Med. 2016; 30(2): 104-113, doi: 10.1007/s12149-015-1037-8, indexed in Pubmed: 26546334.

19. Chang MC, Chen JH, Liang JA, et al. 18F-FDG PET or PET/CT for detection of metastatic lymph nodes in patients with endometrial cancer: A systematic review and meta-analysis. Eur J Radiol. 2012; 81(11): 3511-3517, doi: 10.1016/j.ejrad.2012.01.024.

20. Kitajima K, Murakami K, Yamasaki E, et al. Accuracy of 18F-FDG PET/CT in detecting pelvic and paraaortic lymph node metastasis in patients with endometrial cancer. AJR Am J Roentgenol. 2008; 190(6): 1652-1658, doi: 10.2214/AJR.07.3372, indexed in Pubmed: 18492920.

21. Kitajima K, Murakami K, Yamasaki E, et al. Accuracy of integrated FDG-PET/contrast-enhanced CT in detecting pelvic and paraaortic lymph node metastasis in patients with uterine cancer. Eur Radiol. 2009; 19(6): 1529-1536, doi: 10.1007/s00330-008-1271-8, indexed in Pubmed: 19184037.

22. Suzuki R, Miyagi E, Takahashi N, et al. Validity of positron emission tomography using fluoro-2-deoxyglucose for the preoperative evaluation of endometrial cancer. Int J Gynecol Cancer. 2007; 17(4): 890-896, doi: 10.1111/j.1525-1438.2007.00859.x, indexed in Pubmed: 17343574 